\title{
IL-39 Reduces Proliferation and Promotes Apoptosis of Bladder Cancer by Altering the Activity of Cyclin E and Fas
}

\author{
HUAPING XIAO ${ }^{1,2}$, HAMZA ALISIC ${ }^{1}$, BRANDON T. REIMAN ${ }^{1}$, ZULIANG DENG $^{3}$, ZIWEN ZHU ${ }^{2}$, \\ NATHAN T. GIVENS ${ }^{1}$, QIAN BAI ${ }^{2}$, ANDREW TAIT ${ }^{2}$, MARK R. WAKEFIELD ${ }^{2}$ and YUJIANG FANG ${ }^{1,2}$ \\ ${ }^{1}$ Department of Microbiology, Immunology \& Pathology, Des Moines University, Des Moines, IA, U.S.A.; \\ ${ }^{2}$ Department of Surgery, University of Missouri School of Medicine, Columbia, MO, U.S.A. ; \\ ${ }^{3}$ The Affiliated Hospital of Xiangnan University, Chenzhou, P. R. China
}

\begin{abstract}
Background/Aim: This study was designed to investigate the effect of IL-39 on T24 bladder cancer (BC) cell line survival and growth. Materials and Methods: In order to assess the direct effect of IL-39 on survival, proliferation, and apoptosis of T24 BC cells, we utilized a clonogenic survival assay, a cell proliferation assay, and caspase-3 activity kits. Potential proliferative and apoptotic molecular mechanisms were evaluated by RT-PCR. Results: Treatment of T24 BC cells with IL-39 resulted in a significant reduction in the percentage of colonies. The antitumor effect of IL-39 on T24 bladder cancer cells correlated strongly with a decrease in cyclin $E$, in combination with an increase in the mRNA levels of Fas. Conclusion: IL-39 impedes the growth and survival of T24 BC cells by inhibiting growth and promoting apoptosis. This ability to modulate gene transcription in neoplastic cells shows promise and warrants further research in immunotherapy.
\end{abstract}

According to the American Cancer Society, in 2020 there were approximately 81,400 incidences and 17,980 mortalities involving bladder cancer (1). Bladder cancer in women occurs at lower frequencies, but in men it is the fourth most prevalent cancer with the average age of diagnosis being 73 . The fiveyear survival rates of patients with bladder cancer in situ alone, localized, regional, and distant are $96 \%, 70 \%, 36 \%$ and $5 \%$ respectively (1). Urothelial carcinoma, also referred to as transitional cell carcinoma, accounts for $90 \%$ of the total cases of bladder cancer (2). Of this $90 \%$, approximately $70 \%$ are

This article is freely accessible online.

Correspondence to: Dr. Yujiang Fang, Department of Microbiology \& Immunology, Des Moines University, IA 50312, U.S.A. Tel: +1 5152711435, Fax: +1 5152711543, e-mail: yujiang.fang@dmu.edu

Key Words: IL-23p19/EBI3, IL-39, bladder cancer, proliferation, apoptosis. diagnosed with non-muscle invasive bladder cancer (NMIBC). The recurrence rate of NMIBC is $50 \%-70 \%$, and of that percentage, $10 \%-20 \%$ will progress into muscle invasion bladder cancer (MIBC) $(2,3)$. Nearly $66 \%$ of those who advance to MIBC will succumb to the disease within 48 months even with radical treatment (4). Current treatment of $\mathrm{BC}$ depends on the pathological state of the neoplasm at the time of the transurethral resection of bladder tumor (TURBT) as well as staging, according to the tumor-node-metastasis classification system (5). Treatment for NMIBC and MIBC can range from bladder cancer surgery, intravesical therapy, chemotherapy, radiation therapy, immunotherapy and targeted therapy drugs. Treatment options are contingent upon patient staging, and frequently multiple treatments are used in combination to achieve the desired effect (6).

According to current medical research, exploitation of the immune system and cytokines has shown promise as an effective approach to treat those suffering from varying diseases. Further research is warranted given the viability of a novel bladder cancer treatment method that could diminish the current painful and detrimental treatment options, while increasing the survival rates $(7,8)$. Interleukin-39 (IL-39) is the most recent member of the interleukin-12 cytokine family. It is composed of a heterodimeric chain-pairing of alpha IL23p19 and beta EBI3 (9). IL-39 has been studied in various diseases such as systemic lupus erythematosus (SLE). Furthermore, IL-39 in lupus-like mice was observed to produce an immunopathogenic effect by promoting the proinflammatory response (9). A recent study focused on IL-39's effects on a mouse concanavalin A (ConA) induced liver injury model. The results showed that IL-39 increased serum alanine aminotransferase (ALT) and aspartate aminotransferase (AST) levels, inflammatory infiltration, and hepatocyte necrosis. IL39 also increased serum concentrations of interferon- $\gamma$, tumor necrosis factor- $\alpha$, and IL- $17 \alpha$, eliciting a pro-inflammatory state (10). Based on this concept and other studies, IL-39 has demonstrated the potential to regulate the immune system and possibly provide new treatment alternatives. However, IL-39's 
role in immunotherapy and oncology is still vastly unexplored. The purpose of this study was to investigate the direct effects of IL-39 on the growth, survival, and gene alterations of T24 human bladder cancer cells.

\section{Materials and Methods}

Human bladder cancer cell line. T24 bladder cancer (BC) cell line, derived from human transitional cell carcinoma, was supplied by Dr. Longcheng Li from (University of California, San Francisco, CA, USA). T24 BC cells were cultured in Dulbecco's Modified Eagle Medium (DMEM), supplemented with $10 \%$ heat-inactivated fetal bovine serum (FBS) and $1 \%$ penicillin-streptomycin (Invitrogen, Carlsbad, CA, USA) as previously described (11-15). When cells reached $70 \%$ confluency, they were either treated with IL-39 or DMEM medium alone.

Treatment of T24 BC cells with IL-39. Once 70-80\% confluency was achieved, T24 BC cells were treated with either IL-39 (50 ng/ml R\&D Systems, Minneapolis, MN, USA, Cat\# 9990-IL-050) or DMEM for 3 days. The concentration and incubation time for IL-39 were established from our preliminary experiments and prior cytokine studies (11-15).

Clonogenic survival assay. T24 BC cells were divided and counted by hemocytometer following the 3-day incubation period with IL-39. Clonogenic survival assay was carried out by plating 1,000 cells into a Petri dish (Corning, Lowell, MA, USA) in triplicate. Fresh media were added at day 5 . Nine days after incubation, cells were fixed and stained with $0.05 \%$ crystal violet. Colonies were quantified and then expressed as a percentage of total colonies in the controls.

Determination of proliferation using Quick Cell Proliferation Assay Kit. Quick Cell Proliferation Assay Kit (BioVision, Milpitas, CA, USA), as described by the manufacture's protocol (11-15), was utilized to assess T24 bladder cancer cell proliferation.

Reverse transcription-polymerase chain reaction (RT-PCR). T24 BC cells treated with and without IL-39 were washed with PBS and then homogenized in TRI-zol (Invitrogen, Carlsbad, CA, USA). NanoDrop was used to quantify RNA. Reverse transcription was carried out using $1 \mu \mathrm{g}$ of T24 RNA, as described in previous studies (11-15).

Measurement of Caspase-3 activity. Further evaluation of apoptosis in T24 BC cells was accomplished by assessing caspase- 3 activity using caspase-3/CPP32 colorimetric assay kit (BioVision) as described previously (11-15).

Statistics. All experiments were performed two to three times in duplicate. Statistical analysis was conducted using an unpaired twotailed Student's $t$-test. A $p$-value $<0.05$ was considered significant.

\section{Results}

IL-39 inhibits growth and proliferation of T24 BC cells. The direct effects of IL-39 were examined utilizing T24 BC cells. Once cells reached $70-80 \%$ confluency, they were treated with either IL-39 or medium alone for 3 days. Survival was monitored by using clonogenic survival

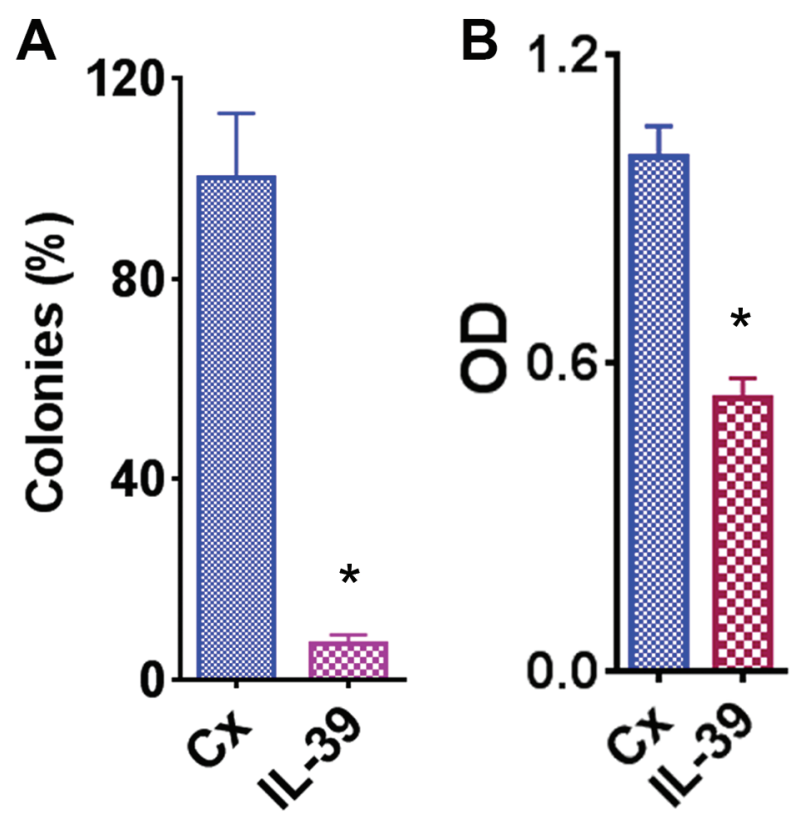

Figure 1. Effect of IL-39 on the growth and proliferation of T24 bladder cancer cells. A: Clonogenic survival assay of T24 bladder cancer cells in the presence or absence of IL-39 $(50 \mathrm{ng} / \mathrm{ml})$. The number of colonies was counted and expressed as a percentage of the total colonies compared with the Control (CX, without IL-39). B: Cell proliferation evaluated using a Cell Proliferation kit in T24 cells treated with IL-39 (50 $\mathrm{ng} / \mathrm{ml})$ or medium alone. Results are expressed as the mean optical density $(O D)+S E M$ of bladder cancer cells in each group. Error bars denote mean $\pm S D$; Student's t-test was used for statistical analysis. Asterisk indicates significant difference $(p<0.05)$.

assays. Considerable differences in growth rate were observed between the two groups. Treatment with IL-39 decreased substantially the growth of T24 BC cells compared to control. This was supported by the reduced OD value measured by Quick Cell Proliferation Assay Kit in the IL-39-treated group when compared to the control (Figure $1, p<0.05)$. These results strongly suggest that IL-39 inhibits proliferation of $\mathrm{T} 24 \mathrm{BC}$ cells.

IL 39 decreases expression of cyclin E in T24 cells. Pro- and anti- proliferative molecules directly control cell proliferation and survival. For example, cyclin B, cyclin D, cyclin E, $\mathrm{cdk} 2$, and cdk4 are crucial pro-proliferative molecules. In contrast, p18, p21, p27, and p53 are essential antiproliferative molecules. mRNA expression of the above stated molecules was examined to determine the direct molecular effects of IL-39 on T24 cell survival. RT-PCR was used to determine mRNA expression levels of the pro- and anti- proliferative molecules in T24 BC cells treated with or without IL-39 $(50 \mathrm{ng} / \mathrm{ml})$. The expression levels of all but two mRNAs were comparable in both groups $(p>0.05)$. 

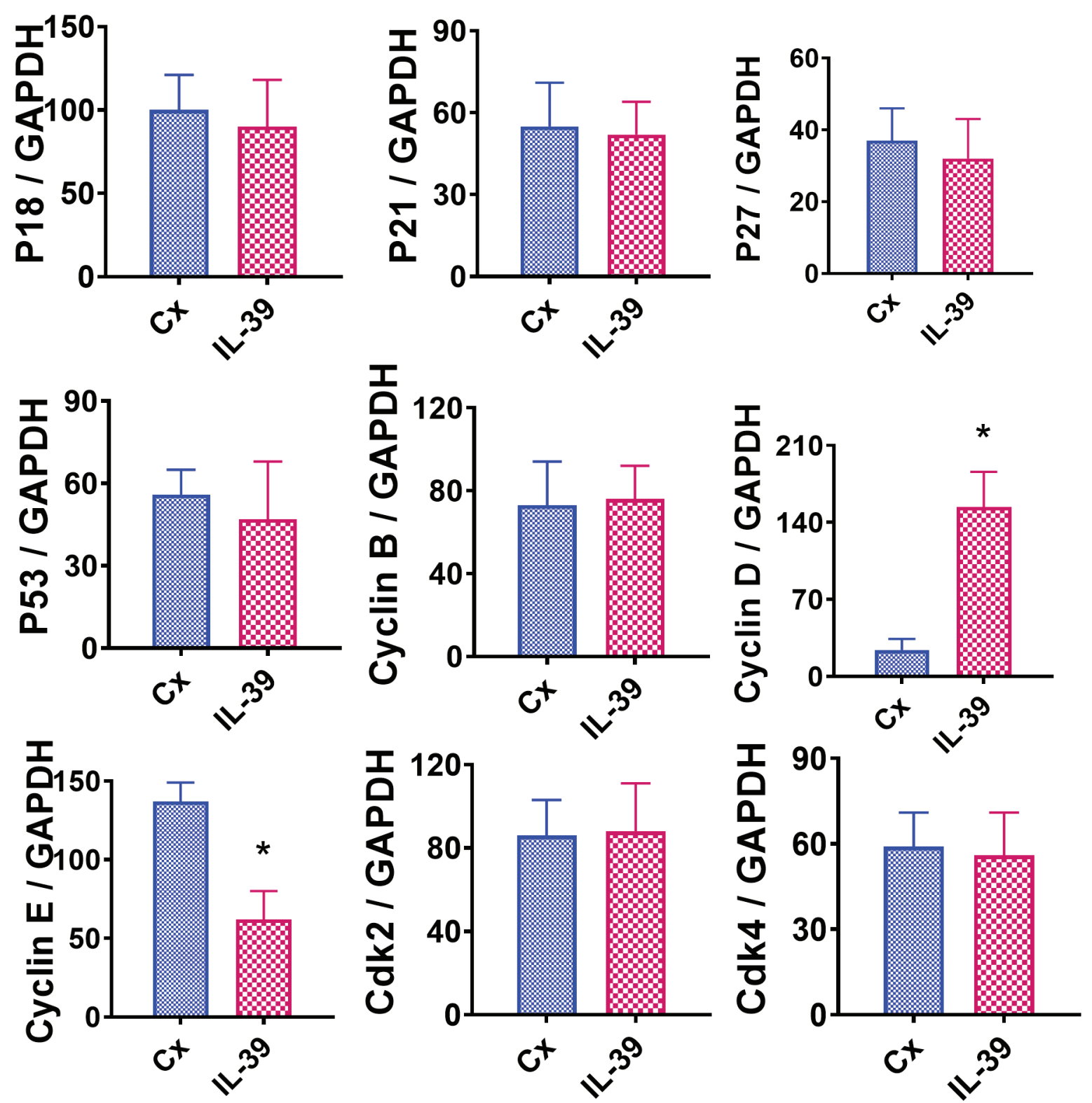

Figure 2. Effect of IL-39 on pro- and anti-proliferative molecules evaluated by RT-PCR. mRNA was extracted as described in the Materials and Methods section. Experiments were performed in triplicate. Results are expressed as the mean ratio of the examined molecule densitometric units/GAPDH + SEM. Significant difference in mRNA expression between cells treated without and with IL-39 is indicated by the asterisk ( $p<0.05)$.

Cyclin D was considerably increased in T24 cells upon treatment with IL-39 compared to the control, whereas cyclin E was significantly decreased in the presence of IL-39 compared to the control (Figure 2; $p<0.05$ ).

IL-39 promotes apoptosis of T24 cells. IL-39's ability to inhibit the proliferation and survival of T24 BC cells may be influenced by an increase in apoptosis. To explore such a pro-apoptotic effect, $70-80 \%$ confluent T24 cells were treated with IL-39 or medium alone for 3 days. Caspase-3 activity kits were then used to assess apoptosis in both the treated cultures and controls. There was a statistically significant $(p<0.05)$ increase in apoptosis in the treated group compared to control (Figure 3 ). This indicates that induction of apoptosis is a secondary mechanism through which IL-39 inhibits T24 BC cell growth. 


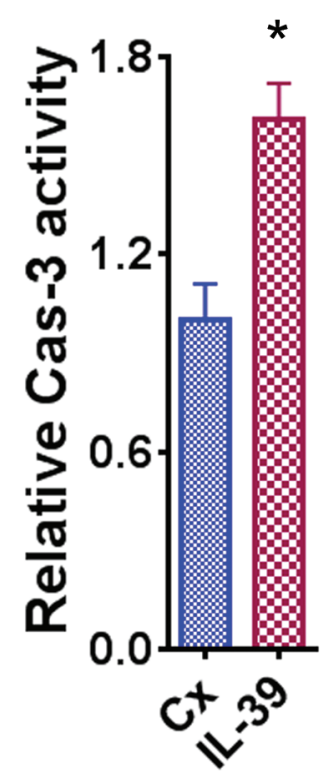

Figure 3. IL-39 promotes apoptosis of T24 bladder cancer cells. T24 cellular caspase-3 activity was measured using caspase-3/CPP32 colorimetric assay kit. Results are expressed as mean activity relative to controls + SEM. Assays were performed in triplicate. Error bars denote mean $\pm S D$; Student's t-test was used for statistical analysis. Asterisk indicates significant difference $(p<0.05)$.

IL-39 up-regulates the expression of the pro-apoptotic molecule Fas in T24 BC cells. The equilibrium between proand anti- apoptotic molecules determines proliferation and senescence, and when altered, it can lead to disease. For instance, Fas, FasL, TRAIL, TRAILR1 and Bax are all notable pro-apoptotic molecules. Conversely, FLIP, Bcl-2 and survivin are essential anti-apoptotic molecules. To investigate the direct effects of IL-39 on apoptosis of T24 $\mathrm{BC}$ cells, the mRNA expression levels of the molecules mentioned above were analyzed using RT-PCR. A prominent increase in the mRNA expression of Fas, a pro-apoptotic molecule, was detected in T24 BC cells treated with IL-39 $(50 \mathrm{ng} / \mathrm{ml})$ compared to the control (Figure 4). This upregulation of the pro-apoptotic molecule Fas indicates an increase in apoptotic activity in $\mathrm{T} 24 \mathrm{BC}$ cells treated with IL-39 $(50 \mathrm{ng} / \mathrm{ml})$.

\section{Discussion}

With more than 80,000 new cases and greater than 17,000 deaths annually in addition to a multitude of undesirable painful treatment options ranging from surgical resection, intravesical chemotherapy, systemic chemotherapy, and radiation therapy, it is understandable why novel treatment options are imperative for patients afflicted by $\mathrm{BC}(1,6)$.
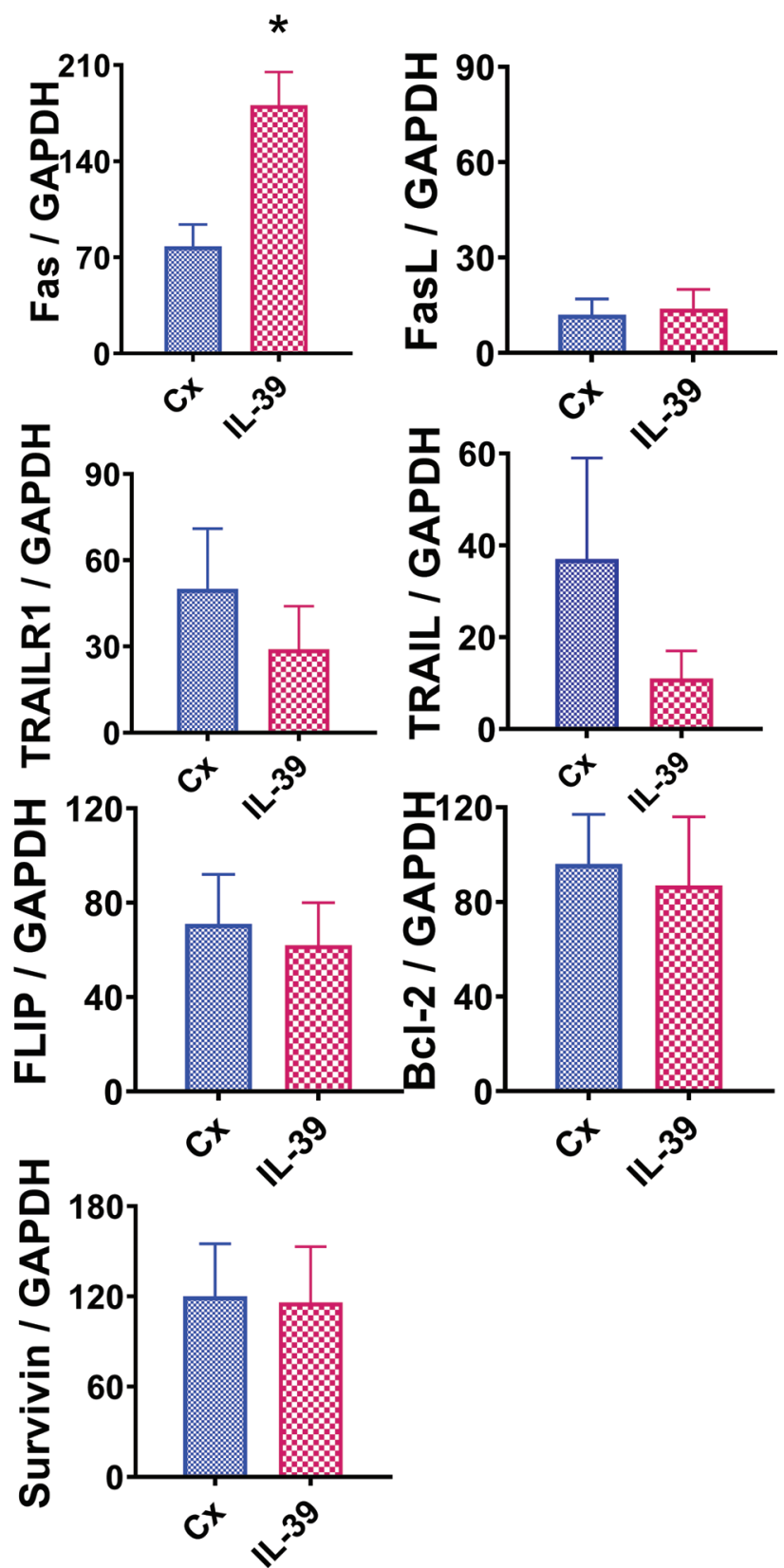

Figure 4. Effect of IL-39 on the expression of pro-and anti-apoptotic molecules as evaluated using RT-PCR. Extraction of mRNA is described in the Materials and Methods section and experiments were performed in triplicate. Results are expressed as the mean ratio of pro- and antiapoptotic molecule densitometric Units/GAPDH + SEM $(\times 100)$. Asterisk indicates a significant difference in mRNA expression between cells treated with IL-39 and untreated (CX) cells.

As immunotherapy expands into more clinical treatment methods and assays are developed for specific BC cell biomarkers such as p53, E-cadherin, bladder tumor antigen 
(BTA) and hyaluronic acid (16), it is logical to explore the potential of cytokines such as IL-39, as treatment modalities.

$\mathrm{BC}$, along with all neoplasms, emerges from mutations that disrupt the cell cycle alongside with mutations that impede a proper immune response. These mutations lead to uninhibited and unchecked growth. For example, cyclin E plays a critical role in the progression from the G1 to the $\mathrm{S}$ phase of the cell cycle. Cyclin E forms a complex with cyclin-dependent kinase, (CDK2) which subsequently phosphorylates retinoblastoma leading to cell cycle progression. Consequently, if a neoplasm up-regulates cyclin E, cell cycle flux will be increased, resulting in a pro-proliferative state. Conversely, Fas is a pro-apoptotic molecule which is often down-regulated in neoplasms. Fas is a member of the tumor necrosis factor (TNF) family. When Fas binds to its adaptor protein receptor, Fas associated death domain (FADD) will be recruited, which will bind procaspase- 8 and form the death inducing signaling complex (DISC). This activates effector caspase-3, ultimately causing apoptosis. If Fas is downregulated, neoplasms can evade apoptosis and grow unchecked. Neoplasms up-regulate or down-regulate a myriad of genes to increase cell proliferation, avoid apoptosis, and promote metastasis. The transitional cell carcinoma explored in this study, T24 cell line, is a common and widely used cell line. The IL-39-induced gene alterations noted in this research will be subsequently compared to other neoplastic cell lines.

Our basic understanding of the pathophysiological role that IL-39 plays in various diseases has been based on prior research. For example, systemic lupus erythematosus research of lupus-like mice found that IL-39 was secreted by stimulated B-cells. B-cell stimulation causes an up-regulation of neutrophils and subsequent a positive feedback loop. This positive feedback loop was found to be mediated by the B cell activation factor, BAFF, which subsequently results in further B-cell activation and IL-39 secretion. The Interleukin-12 family, including IL-39, have demonstrated promise as potential therapeutic targets in autoimmune diseases $(9$, 17). Furthermore, anti-IL-39 polyclonal antibodies in lupus-like mice reduced both inflammatory cell numbers as well as infiltrating inflammatory cells, resulting in a reduction of autoimmune symptoms (18). Additional IL39 research in skin diseases such as psoriasis and atopic dermatitis as well as ConA-induced hepatitis have been shown to play a pro-inflammatory role $(19,20)$. Conversely, IL-39 has been discovered to play a different role in adipose tissue. In adipose tissue, IL-39 has been shown to affect antiinflammatory genes, exhibit a paracrine/autocrine insulinsensitizing action, as well as to stimulate muscle-fat crosstalk to promote the expression of genes that are associated with browning of the white adipose tissue $(21,22)$.

Due to various roles of IL-39 in a tissue non-specific pattern and in the immune system, further investigation of its involvement in different pathophysiological states, particularly bladder cancer, is warranted. In this study, we utilized the T24 BC cell line to investigate the direct effects of IL-39. Notably, we discovered that treatment of T24 BC cells with IL-39 inhibited proliferation while simultaneously promoting apoptosis. These results were supported by substantially decreased percentages of colonies formed by T24 BC cells in the presence of IL-39. In addition, IL-39 increased caspase-3 activity indicating induction of apoptosis. To expand our understanding of BC's uncontrolled growth, we analyzed the expression of proliferation and apoptosis factors. We found an anti-tumor effect of IL-39 on T24 BC cells that correlated with a decrease in the levels of the pro-proliferative molecule cyclin $\mathrm{E}$ and an increase in the pro-apoptotic molecule Fas. Together, these results support IL-39's capacity to exhibit an antiproliferation and pro-apoptotic activity in bladder cancer cells.

An equilibrium between proliferative and apoptotic molecules is present in healthy individuals, that when unbalanced can lead to various diseases. The molecules that promote cell proliferation include cyclin B, cyclin D, cyclin $\mathrm{E}$, cdk2 and cdk4, whereas the anti-proliferative molecules include p18, p21, p27 and p53 (23-25). Similarly, proapoptotic molecules include Fas, FasL, TRAIL, and TRAILR1, whereas anti-apoptotic molecules include FLIP, Bcl-2 and survivin (26). Our results demonstrated that IL-39 affects $\mathrm{BC}$ by decreasing the levels of the pro-proliferative molecule cyclin E, while increasing those of the pro-apoptotic molecule Fas (26). The levels of Cyclin D, a pro-proliferative molecule, were also found to be up-regulated. This could be a protective factor to compensate for the decrease seen in cyclin E. Thus, our results revealed IL-39's capacity to shift the proliferative and apoptotic molecular equilibrium in T24 BC cells. This shift ultimately causes decreased cell proliferation and increased apoptosis. IL-39's ability to regulate gene transcription in neoplastic cells shows promise and warrants further research in immunotherapy.

There are several limitations to this study. First, it was carried out at the mRNA level and was not reinforced by protein analysis techniques such as immunohistochemical staining (IHC). This would have confirmed that the upregulation observed in mRNA did result in increased translation of the corresponding proteins. Furthermore, our experiment focused on one cell line, T24 BC, which limits our ability to generalize our results to other bladder cancers. Currently, IL-39's effects on various cell lines, including other cell lines of BC, are being explored in our lab. We are also implementing IHC staining to reinforce the upregulation seen in the mRNA levels.

\section{Conflicts of Interest}

The Authors have no conflicts of interest to declare regarding this study. 


\section{Authors' Contributions}

YF conceived and designed this study. All Authors performed experiments. YF and $\mathrm{ZZ}$ analyzed and interpreted the data. HX and YF wrote the draft and made critical revision. All Authors approved the final version of the article.

\section{Acknowledgements}

This work was supported by grants from Des Moines University (Des Moines, IA, USA) to Yujiang Fang. ZD was supported by The Key Project of Education Department of Hunan Province (No. 19A458).

\section{References}

1 Siegel RL, Miller KD and Jemal A: Cancer statistics, 2020. CA Cancer J Clin 70(1): 7-30, 2020. PMID: 31912902. DOI: $10.3322 /$ caac 21590

2 Bellmunt J, Orsola A, Leow JJ, Wiegel T, De Santis M, Horwich A and ESMO Guidelines Working Group: Bladder cancer: ESMO practice guidelines for diagnosis, treatment and followup. Ann Oncol 25(Suppl 3): iii40-iii48, 2014. PMID: 25096609. DOI: $10.1093 /$ annonc/mdu223

3 Martinez Rodriguez RH, Buisan Rueda $\mathrm{O}$ and Ibarz L: Bladder cancer: Present and future. Med Clin (Barc) 149(10): 449-455, 2017. PMID: 28736063. DOI: 10.1016/j.medcli. 2017.06.009.

4 van den Bosch S and Alfred Witjes J: Long-term cancer-specific survival in patients with high-risk, non-muscle-invasive bladder cancer and tumour progression: A systematic review. Eur Urol 60(3): 493-500, 2011. PMID: 21664041. DOI: 10.1016/j.eururo. 2011.05 .045

5 Sharma S, Ksheersagar $\mathrm{P}$ and Sharma P: Diagnosis and treatment of bladder cancer. Am Fam Physician 80(7): 717-723, 2009. PMID: 19817342.

6 Zhang J, Wang Y, Weng H, Wang D, Han F, Huang Q, Deng T, Wang $X$ and Jin Y: Management of non-muscle-invasive bladder cancer: Quality of clinical practice guidelines and variations in recommendations. BMC Cancer 19(1): 1054, 2019. PMID: 31694589. DOI: 10.1186/s12885-019-6304-y

7 Porta C, Paglino C, Imarisio I and Bonomi L: Cytokine-based immunotherapy for advanced kidney cancer: Past results and future perspectives in the era of molecularly targeted agents. ScientificWorldJournal 7: 837-849, 2007. PMID: 17619768. DOI: $10.1100 /$ tsw.2007.154

8 Conlon KC, Miljkovic MD and Waldmann TA: Cytokines in the treatment of cancer. J Interferon Cytokine Res 39(1): 6-21, 2019. PMID: 29889594. DOI: 10.1089/jir.2018.0019

9 Wang X, Wei Y, Xiao H, Liu X, Zhang Y, Han G, Chen G, Hou C, Ma N, Shen B, Li Y, Egwuagu CE and Wang R: A novel IL23p19/Ebi3 (IL-39) cytokine mediates inflammation in Lupuslike mice. Eur J Immunol 46(6): 1343-1350, 2016. PMID: 27019190. DOI: 10.1002/eji.201546095

10 Li Y, Gong L, Weng L, Pan X, Liu C and Li M: Interleukin-39 exacerbates concanavalin A-induced liver injury. Immunopharmacol Immunotoxicol 43(1): 94-99, 2021. PMID: 33412981. DOI: 10.1080/08923973.2020.1869778

11 Fang Y, Chen X, Bai Q, Qin C, Mohamud AO, Zhu Z, Ball TW, Ruth CM, Newcomer DR, Herrick EJ and Nicholl MB: IL-9 inhibits HTB-72 melanoma cell growth through upregulation of p21 and TRAIL. J Surg Oncol 111(8): 969-974, 2015. PMID: 25988864. DOI: $10.1002 /$ jso.23930

12 Zhu Z, Davidson KT, Brittingham A, Wakefield MR, Bai Q, Xiao $\mathrm{H}$ and Fang Y: Trichomonas vaginalis: A possible foe to prostate cancer. Med Oncol 33(10): 115, 2016. PMID: 27613161. DOI: 10.1007/s12032-016-0832-y

13 Ding VA, Zhu Z, Steele TA, Wakefield MR, Xiao H, Balabanov $\mathrm{D}$ and Fang Y: The novel role of IL-37 in prostate cancer: Evidence as a promising radiosensitizer. Med Oncol 35(1): 6, 2017. PMID: 29210005. DOI: 10.1007/s 12032-017-1070-7

14 Fang Y, Zhao L, Xiao H, Cook KM, Bai Q, Herrick EJ, Chen X, Qin C, Zhu Z, Wakefield MR and Nicholl MB: IL-33 acts as a foe to MIA PaCa-2 pancreatic cancer. Med Oncol 34(2): 23, 2017. PMID: 28058630. DOI: 10.1007/s12032-016-0880-3

15 Chen X, Lu K, Timko NJ, Weir DM, Zhu Z, Qin C, Mann JD, Bai Q, Xiao H, Nicholl MB, Wakefield MR and Fang Y: IL-33 notably inhibits the growth of colon cancer cells. Oncol Lett 16(1): 769-774, 2018. PMID: 29963144. DOI: 10.3892/ol. 2018.8728

16 Stefan-van Staden RI, Gheorghe DC, Jinga V, Sima CS and Geanta M: Fast screening of whole blood and tumor tissue for bladder cancer biomarkers using stochastic needle sensors. Sensors (Basel) 20(8): 2420, 2020. PMID: 32344622. DOI: $10.3390 / \mathrm{s} 20082420$

17 Wang X, Liu X, Zhang Y, Wang Z, Zhu G, Han G, Chen G, Hou C, Wang T, Ma N, Shen B, Li Y, Xiao H and Wang R: Interleukin (IL)-39 [IL-23p19/Epstein-Barr virus-induced 3 (Ebi3)] induces differentiation/expansion of neutrophils in lupusprone mice. Clin Exp Immunol 186(2): 144-156, 2016. PMID: 27400195. DOI: $10.1111 /$ cei.12840

18 Wang X, Zhang Y, Wang Z, Liu X, Zhu G, Han G, Chen G, Hou C, Wang T, Shen B, Li Y, Xiao H, Ma N and Wang R: Anti IL 39 (IL 23p19/Ebi3) polyclonal antibodies ameliorate autoimmune symptoms in lupus like mice. Mol Med Rep 17(1): 1660-1666, 2018. PMID: 29138852. DOI: $10.3892 / \mathrm{mmr} .2017 .8048$

19 Wechter T, Cline A and Feldman SR: Targeting p19 as a treatment option for psoriasis: An evidence-based review of guselkumab. Ther Clin Risk Manag 14: 1489-1497, 2018. PMID: 30174431. DOI: 10.2147/TCRM.S177127

20 Ushach I, Burkhardt AM, Martinez C, Hevezi PA, Gerber PA, Buhren BA, Schrumpf H, Valle-Rios R, Vazquez MI, Homey B and Zlotnik A: Meteorin-like is a cytokine associated with barrier tissues and alternatively activated macrophages. Clin Immunol 156(2): 119-127, 2015. PMID: 25486603. DOI: 10.1016/j.clim.2014.11.006

21 Zheng SL, Li ZY, Song J, Liu JM and Miao CY: Metrnl: A secreted protein with new emerging functions. Acta Pharmacol Sin 37(5): 571-579, 2016. PMID: 27063217. DOI: 10.1038/aps.2016.9

22 Li ZY, Song J, Zheng SL, Fan MB, Guan YF, Qu Y, Xu J, Wang $\mathrm{P}$ and Miao CY: Adipocyte metrnl antagonizes insulin resistance through PPAR $\gamma$ signaling. Diabetes 64(12): 4011-4022, 2015. PMID: 26307585. DOI: 10.2337/db15-0274

23 Lenz P, Pfeiffer R, Baris D, Schned AR, Takikita M, Poscablo MC, Schwenn M, Johnson A, Jones M, Kida M, Cantor KP, Rothman N, Silverman DT, Hewitt SM and Moore LE: Cellcycle control in urothelial carcinoma: Large-scale tissue array analysis of tumor tissue from Maine and Vermont. Cancer Epidemiol Biomarkers Prev 21(9): 1555-1564, 2012. PMID: 22761304. DOI: 10.1158/1055-9965.EPI-12-0261 
24 Fang $\mathrm{Y}$, Yu S and Braley-Mullen H: TGF- $\beta$ promotes proliferation of thyroid epithelial cells in IFN- $\gamma(-/-)$ mice by down-regulation of p21 and p27 via AKT pathway. Am J Pathol 180(2): 650-660, 2012. PMID: 22119715. DOI: 10.1016/ j.ajpath.2011.10.009

25 Zhu Z, Achreja A, Meurs N, Animasahun O, Owen S, Mittal A, Parikh P, Lo TW, Franco-Barraza J, Shi J, Gunchick V, Sherman MH, Cukierman E, Pickering AM, Maitra A, Sahai V, Morgan MA, Nagrath S, Lawrence TS and Nagrath D: Tumourreprogrammed stromal BCAT1 fuels branched-chain ketoacid dependency in stromal-rich PDAC tumours. Nat Metab 2(8): 775-792, 2020. PMID: 32694827. DOI: 10.1038/s42255-0200226-5
26 Greil R, Egle A and Villunger A: On the role and significance of Fas (Apo-1/CD95) ligand (FasL) expression in immune privileged tissues and cancer cells using multiple myeloma as a model. Leuk Lymphoma 31(5-6): 477-490, 1998. PMID: 9922038. DOI: 10.3109/10428199809057607

Received March 26, 2021

Revised April 2, 2021

Accepted April 5, 2021 